\title{
Periprocedural and clinical outcomes of percutaneous coronary intervention of chronic total occlusions in patients with low- and mid-range ejection fractions
}

Waleed Salem El Awady ${ }^{1}$, Mohamed Samy ${ }^{1 *}$ (D), Mohammad Mustafa Al-Daydamony', Magdy Mohammad Abd El Samei ${ }^{1}$ and Khaled Abd El Azim Shokry ${ }^{2}$

\begin{abstract}
Background: The benefit and safety of percutaneous coronary intervention (PCI) to chronic total occlusions (CTO) in patients with low and mid-range left ventricular ejection fraction (LVEF) continue to be evidence limited. The aim of our study was to investigate the impact of LVEF in patients undergoing CTO PCI and to evaluate the mid-term clinical outcome of those with low and mid-range LVEF. We assessed the periprocedural and mid-term outcomes of 75 patients undergoing CTO PCls according to pre-procedural LVEF: group (N), $\geq 50 \%$ (normal, nom.= 25); group (M), 40-49\% (mid-range, nom.= 25); and group $(L),<40 \%$ (low, nom.= 25); within 6 months of follow-up.
\end{abstract}

Results: The prevalence of DM and chronic kidney disease (CKD) was significantly higher in low LVEF group (60\%, $p=0.04$ and $48 \%, p=0.01$ respectively). Apart from significantly lower contrast volume in patients with low LVEF ( $p=0.04$ ), there was no significant difference between the three groups regarding the procedural time, SYNTAX score and J-CTO score. We noticed similar procedural success in the three groups ( $88 \%$ vs. $84 \%$ vs. $76 \%, p=0.521$ ). LVEF category failed to predict procedural success ( $O R=0.652, p=0.268)$. There was a highly significant improvement in angina 6 months following intervention in normal LVEF group ( $p$ value $<0.001$ ). Grade of dyspnea significantly improved 6 months following intervention in mid-range LVEF and low LVEF groups ( $p$ value $=0.04$ and 0.031 respectively). There was no significant difference between the three groups regarding the reported MACCEs (12\% vs. $16 \%$ vs. $28 \%, p=0.268)$.

Conclusion: $\mathrm{CTO} P \mathrm{PCl}$ represents an efficient and safe strategy in patients with low and mid-range LVEFs. Mid-term outcomes in these patients were significantly improved following successful CTO PCl, without higher risk of MACCE at 6 months follow-up.

Keywords: Chronic total occlusion, Percutanous coronary intervention, Left ventricular ejection fraction

\section{Background}

Coronary chronic total occlusions (CTOs) are described as an occluded coronary artery with thrombolysis in myocardial infarction (TIMI) flow 0 for a period exceeding 3 months. Euro CTO club consensus stratified the

\footnotetext{
* Correspondence: mohammedsamyabdulaziz87@gmail.com

${ }^{1}$ Cardiology Department, Faculty of Medicine, Zagazig University, Zagazig,

Ash Sharkia, Egypt

Full list of author information is available at the end of the article
}

obstruction duration into 3 grades: (I) "certain" (confirmed by angiography), if TIMI 0 flow was confirmed by an angiogram done more than 3 months ago; (II) "likely" (confirmed by clinical data), if an acute myocardial infarction (MI) occurred more than 3 months ago in the occluded artery territory with no other culprits incriminated in the current angiography; (III) "undetermined", TIMI 0 flow and angiographically anatomical configuration suggesting long-standing occlusion with 
stable angina unchanged in the last 3 months or evidence of silent ischemia [1].

CTOs are one of the most challenging targets of percutaneous coronary intervention (PCI), due to their complexity, with lower procedural success rates and greater complication rates, radiation exposure and procedure times compared with non-CTO PCI. Indeed, in stable multivessel coronary artery disease (CAD), CTO was the most robust independent predictor of failed PCI, and was associated with worse prognosis [2]. However, recent advances in the interventional techniques, dedicated equipment and CTO expertise have resulted in > $90 \%$ procedural success rates, and lower major complications [3]. Reported benefits of successful CTO PCI included improved symptoms, ventricular function, and survival [4].

Left ventricular ejection fraction (LVEF) is a well established independent prognostic factor in patients with CAD [5]. Severe ischemic left ventricular dysfunction is related to higher morbidity, an increased risk of sudden death due to ventricular arrhythmias, poor quality of life, and frequent re-hospitalization for heart failure, with CTO patients having more comorbidities and procedural drawbacks [6].

Data regarding procedural success and clinical outcomes of CTO PCI is limited throughout literature, in patients with low $(<40 \%)$ and mid-range LVEF (40$49 \%)$. The aim of our study was to assess the impact of LVEF in patients undergoing CTO PCI and to evaluate the mid-term clinical outcome of those with low and mid-range LVEF.

\section{Methods}

\section{Study design}

This prospective study was accomplished from November (2017) to April (2019). We included 75 patients coming to the catheterization laboratory unit to undergo elective CTO PCI.

The procedure was well explained to each patient and a detailed informed verbal consent was obtained. The study protocol was approved by our Institutional Clinical Research and Ethics Committee.

\section{Patient selection}

Selected patients had at least one epicardial coronary artery CTO proved by former coronary angiography and indicated for PCI; i.e., presence of viable myocardium in the supplied territory in the presence of significant ischemia and/or presence of refractory angina [Class III/ IV\} or new crescendo angina not explained by any other lesions $[7,8]$.

We excluded patients with acute or recent coronary occlusions, recent revascularization in the past 3 months, non-viable myocardium, previous failure of CTO PCI, poor echocardiographic window, significant structural heart disease, decompensated heart failure, and active infections.

We subdivided the study population into 3 groups based on LVEF: group (N), normal LVEF ( $\geq 50 \%$ ); group (M), mid-range LVEF (40-49\%); and group (L), low $\operatorname{LVEF}(<40 \%)$.

Every patient was assessed by full history analysis and thorough clinical examination. Laboratory analysis of serum creatinine, urea, liver function test, fasting and postprandial blood glucose, and lipid profile was performed. Twelve-lead ECG was assessed for evidence of ischemic heart disease (IHD) or prior MI. Baseline angina and dyspnea were graded according to Canadian Cardiovascular Society (CCS) [9] and New York Heart Association (NYHA) classes [10] respectively, prior to the CTO PCI, then reassesed 6 months post-PCI.

\section{Echocardiography}

Every patient underwent echocardiography within $24 \mathrm{~h}$ before intervention, based on the recommendation of the American Society of Echocardiography [11, 12]. The echocardiographic examination was accomplished using General Electric Vivid 7 (Norway) and Siemens Acuson NC 1000 (Germany) machines, using 2 to 2.5 MHZ transthoracic transducers. Echocardiograms were performed in the supine and/or left lateral position. Modified biplane Simpson's method was used to assess LVEF [11].

Patients with impaired LVEF and without significant angina and/or new crescendo angina underwent lowdose dobutamine stress echocardiography (DSE) for assessment of contractile reserve. Hypokinetic or akinetic myocardial segments that showed improved contractility with low-dose DSE were considered viable [12].

\section{Coronary intervention}

We used General Electric and SIEMENS catheterization systems for our procedures. CTOs were defined as an evidence of total occlusions in a major epicardial coronary artery ( $\geq 2.5 \mathrm{~mm}$ in diameter), with durations $\geq 3$ months [1]. CTO lesions complexity and CTO PCI attempt difficulty were evaluated based on the J-CTO (Japanese multicenter registry) score [13]. Syntax score was used to evaluate the coronary lesions [14]. Coronary angioplasty was done for CTO lesions; with access site, techniques of wiring, and dedicated tools left for operators' preferences. Interventions resulting in reduction of luminal diameter of less than $20 \%$ compared to the adjacent normal coronary vessel were considered successful. Interventions that could not achieve at least TIMI 2 flow were considered failed $[15,16]$. Patients were prescribed guideline-derived optimal medical treatment, and a follow-up for 6 months was done. 


\section{Clinical outcome and follow up endpoints}

Procedural success was defined as angiographically successful revascularization without procedure-related complications, including cardiac death, acute MI, perforation with or without tamponade, access site complications, and contrast-induced nephropathy (CIN). Major bleeding was assessed according to TIMI bleeding classification [17]. Major adverse cardiac and cerebrovascular events (MACCE) were defined as the composite of cardiac death, MI, acute heart failure, stroke, CTO target vessel revascularization (TVR) and non-TVR, and assessed within 6 months of follow-up post CTO PCI.

\section{Statistical analysis}

Data collected throughout history, basic clinical characteristics, and outcome measures; were coded and entered using the Microsoft Excel software, then imported into the Statistical Package for the Social Sciences (SPSS version 20.0) software for analysis. Chi-square and Fisher exact tests were used to compare categorical data. Continuous data were summarized as mean \pm standard deviation (SD). One way ANOVA test was used for comparison of LVEF in the three groups. Paired sample $t$ test was performed to assess the difference between means \pm SDs of one variable before and after intervention. Finally, independent predictors for procedural success were identified using univariate and multivariate binary logistic regression analyses models.

\section{Results}

The study population had a mean age of $60.51 \pm 8.09$ years and a mean BMI of $27.68 \pm 2.1 .60$ patients $(80 \%)$ were males, 58 patients $(77.3 \%)$ had hypertension, 47 patients $(62.7 \%)$ had dyslipidemia, 53 patients $(70.7 \%)$ were current smokers, while CKD was recorded in 20 patients (26.7\%).
Group (L) showed a significantly higher prevalence of CKD and DM $(p<0.05)$. However, the 3 study groups showed no significant difference as regard the age, BMI, dyslipidemia, gender, hypertension, history of previous revascularization, or smoking $(p>0.05)$ (Table 1). Clinically, patients with advanced exertional chest pain of CCS class $\geq 3$ were significantly higher in group $(\mathrm{N})\left(\chi^{2}\right.$ $=9.301, p<0.05)$, while exertional dyspnea of NYHA class $\geq 3$ was significantly higher in group $(\mathrm{L})\left(\chi^{2}=\right.$ 12.052, $p<0.05$ ) (Table 2).

Apart from contrast volume that was significantly lower in patients with low LVEF $(\mathrm{F}=2.95, \mathrm{p}<0.05)$, there was no significant difference between the three groups regarding the procedural time, SYNTAX score, and J-CTO score. Antegrade wiring technique was successful in 57 (76\%) out of the 75 patients, with no statistically significant difference among the three groups $(\mathrm{p}<$ $0.05)$. Guidewires of $<3 \mathrm{gm}$ tip load were used in 48 (64\%) out of 75 patients, with almost same percentage among the three groups $(\mathrm{p}>0.05)$ (Table 3$)$.

Procedural success was recorded in $22(88 \%)$ of 25 patients of group $(\mathrm{N})$, while periprocedural complications were determined in 3 cases ( 1 case of NSTE-ACS, 1 case of non-flow limiting dissection and 1 case of self-limited coronary perforation without tamponade). In group (M), procedural success was recorded in $21(84 \%)$ of 25 patients, while periprocedural complications were determined in 4 cases ( 1 case of AF, 1 case of heart failure, 1 case of coronary perforation with tamponade, and 1 case of non-major vascular site complication). In group (L), procedural success was recorded in 19 (76\%) of 25 patients, while periprocedural complications were determined in 6 cases ( 3 cases of CIN, 2 cases of heart failure, and 1 case of AF). There was no significant difference in the procedural success between the three groups $\left(\chi^{2}=\right.$ 1.303 and $p>0.05$ ).

Table 1 Patient characteristics and risk factors in the study groups

\begin{tabular}{|c|c|c|c|c|}
\hline Variable & G (N) (nom. = 25) & G (M) (nom. = 25) & $G(L)(n o m .=25)$ & $p$ value \\
\hline Age (years) (mean $\pm S D$ ) & $60.40 \pm 8.37$ & $60.042 \pm 7.91$ & $61.07 \pm 8.24$ & 0.901 \\
\hline BMI $\left(\mathrm{kg} / \mathrm{m}^{2}\right)($ mean $\pm \mathrm{SD})$ & $27.69 \pm 2.44$ & $27.81 \pm 2.21$ & $27.58 \pm 1.64$ & 0.928 \\
\hline CKD (nom. (\%)) & $3(12 \%)$ & $5(20 \%)$ & $12(48 \%)$ & $0.01^{*}$ \\
\hline DM (nom. (\%)) & $7(28 \%)$ & $8(32 \%)$ & $15(60 \%)$ & $0.042^{*}$ \\
\hline Dyslipidemia (nom. (\%)) & $14(56 \%)$ & $16(64 \%)$ & $17(68 \%)$ & 0.671 \\
\hline Hypertension (nom. (\%)) & $20(80 \%)$ & $18(72 \%)$ & $20(80 \%)$ & 0.738 \\
\hline Male (nom. (\%)) & $21(84 \%)$ & $20(80 \%)$ & $19(76 \%)$ & 0.779 \\
\hline Post CABG (nom. (\%)) & $3(12 \%)$ & $5(20 \%)$ & $4(16 \%)$ & 0.743 \\
\hline Prior PCI (nom. (\%)) & $8(32 \%)$ & $10(40 \%)$ & $5(20 \%)$ & 0.304 \\
\hline Smoking (nom. (\%)) & $16(64 \%)$ & 17 (68\%) & $20(80 \%)$ & 0.433 \\
\hline
\end{tabular}

Data are expressed as mean \pm SD or number (\%)

$B M I$ : body mass index, CABG: coronary artery bypass graft, CKD: chronic kidney disease, DM: diabetes mellitus, $G(N)$ : normal LVEF group, $G(M)$ : mid-range LVEF group, $G(L)$ : low LVEF group, *:significant 
Table 2 Baseline clinical data in the 3 groups

\begin{tabular}{llll}
\hline Variable & $G(N)($ nom. $=25)$ & $G(M)($ nom. $=25)$ & $G(L)($ nom. $=25)$ \\
Nom. $(\%)$ & Nom. $(\%)$ & $\begin{array}{l}p \\
\text { value }\end{array}$ \\
\hline CCS class & & $13(52 \%)$ \\
1,2 & $3(12 \%)$ & $10(40 \%)$ & $12(48 \%)$ \\
3,4 & $22(88 \%)$ & $15(60 \%)$ & $7(28 \%)$ \\
NYHA class & & $18(72 \%)$ \\
1,2 & $19(76 \%)$ & $15(60 \%)$ & $0.01^{*}$ \\
3,4 & $6(24 \%)$ & $10(40 \%)$ & $0.002^{*}$ \\
\hline
\end{tabular}

Data are expressed as numbers (\%)

CCS: Canadian Cardiovascular Society; $G(N)$ : normal LVEF group, G $(M)$ : mid-range LVEF group, G (L): low LVEF group; NYHA: New York Heart Association, *:significant

By applying univariate then multivariate logistic regression analysis models, only DM and J-CTO scores were proved to be independent predictors of procedural success; where periprocedural complications were more reported in diabetic patients and those with J-CTO score $>3(\mathrm{OR}=4.884$ and 15.882 respectively, $p<0.05)$. All other factors, including LVEF category failed to predict procedural success (Table 4).

Clinically, regarding angina follow up, each group was subdivided into 2 subgroups: angina I (patients with no angina or CCS class 1 or 2) and angina II (patients with CCS class $\geq 3$ ). In group $(\mathrm{N})$, there was a significant improvement in angina 6 months post PCI $($ McNemar $=$ 0.01., $p$ value $<0.001$ ). Despite angina improvement was evident in the other 2 groups, yet without statistical significance $(p>0.05)$ (Fig. 1).

In the same context, each group was subdivided into 2 subgroups regarding dyspnea grades: dyspnea I (patients with no dyspnea or NYHA class 1 or 2) and dyspnea II (patients with NYHA class $\geq 3$ ). There was a significant improvement in the grade of dypnea 6 months post CTO PCI, in group (M) $(\mathrm{McNemar}=3.841, p$ value $<$ $0.05)$ and group $(\mathrm{L})(\mathrm{McNemar}=8.615, p$ value $<0.05)$ (Fig. 2).

All patients of the 3 study groups were assessed during the period of 6 months follow-up for MACCE. There was no mortality cases recorded in any group, whereas MACCE were recorded in 14 (18.6\%) out of 75 cases. In

Table 3 Angiographic characteristics and procedural data in the 3 groups

\begin{tabular}{|c|c|c|c|c|}
\hline Variable & $\mathrm{G}(\mathrm{N})(\mathrm{nom} .=25)$ & $\mathrm{G}(\mathrm{M})(\mathrm{nom} .=25)$ & $\mathrm{G}(\mathrm{L})(\mathrm{nom} .=25)$ & $p$ value \\
\hline \multicolumn{5}{|c|}{ Procedure time (min) } \\
\hline Mean \pm SD & $79.12 \pm 30.24$ & $80.36 \pm 27.54$ & $80.28 \pm 25.32$ & 0.985 \\
\hline Range & $35-150$ & $36-145$ & $40-130$ & \\
\hline \multicolumn{5}{|c|}{ Contrast volume $(\mathrm{ml})$} \\
\hline Mean \pm SD & $357.3 \pm 215.2$ & $339.8 \pm 198.4$ & $276.4 \pm 139.4$ & $0.04^{*}$ \\
\hline Range & $142-573$ & $141-538$ & $137-415$ & \\
\hline \multicolumn{5}{|l|}{ Syntax score } \\
\hline Mean \pm SD & $21.64 \pm 3.90$ & $22.72 \pm 3.35$ & $22.84 \pm 2.56$ & 0.376 \\
\hline Range & $15-30$ & $17-29$ & $19-29$ & \\
\hline \multicolumn{5}{|c|}{ J-CTO score (N. (\%)) } \\
\hline$\geq 3$ & $12(48 \%)$ & $13(52 \%)$ & 15 (60\%) & 0.687 \\
\hline$<3$ & $13(52 \%)$ & 12 (48\%) & $10(40 \%)$ & \\
\hline \multicolumn{5}{|c|}{ Wire technique } \\
\hline Antegrade & $20(80 \%)$ & $19(76 \%$ & $18(72 \%)$ & 0.803 \\
\hline Retrograde & $5(20 \%)$ & $6(24 \%)$ & $7(28 \%)$ & \\
\hline \multicolumn{5}{|c|}{ Wire stiffness } \\
\hline$<3 \mathrm{~g}$ & 17 (68\%) & 15 (60\%) & $16(64 \%)$ & 0.841 \\
\hline$>3 \mathrm{~g}$ & $8(32 \%)$ & $10(40 \%)$ & $9(36 \%)$ & \\
\hline
\end{tabular}

Data are expressed as mean \pm SD or number (\%)

$G(N)$ : normal LVEF group, $G(M)$ : mid-range LVEF group, $G(L)$ : low LVEF group, *:significant 
Table 4 Univariate and multivariate logistic regression analyses for predictors of procedural success

\begin{tabular}{|c|c|c|c|c|c|c|c|}
\hline Variable & Exp (B) odds ratio & $95 \% \mathrm{Cl}$ & $p$ value & Variable & Exp (B) odds ratio & $95 \% \mathrm{Cl}$ & $p$ value \\
\hline \multicolumn{8}{|c|}{ Univariate logistic regression analysis } \\
\hline DM & 4.393 & $1.209 / 15.957$ & $0.025^{*}$ & Prior PCl & 1.528 & $0.440 / 5.304$ & 0.505 \\
\hline EF category & 0.652 & $0.305 / 1.389$ & 0.268 & CKD & 0.444 & $0.089 / 2.209$ & 0.322 \\
\hline Age & 1.055 & $0.975 / 1.142$ & 0.182 & Post CABG & 1.767 & $0.406 / 7.692$ & 0.448 \\
\hline Gender & 0.485 & $0.126 / 1.865$ & 0.292 & SYNTAX score & 0.905 & $0.756 / 1.084$ & 0.279 \\
\hline BMl & 0.909 & $0.689 / 1.197$ & 0.496 & J-CTO score & 14.571 & $1.784 / 119.043$ & $0.012^{*}$ \\
\hline HTN & 0.384 & $0.106 / 1.385$ & 0.144 & Wiring technique & 0.658 & $0.175 / 2.456$ & 0.532 \\
\hline Smoking & 0.604 & $0.173 / 2.107$ & 0.429 & Wiring stiffness & 1.327 & $0.367 / 4.799$ & 0.666 \\
\hline Dislipidemia & 0.642 & $0.192 / 2.148$ & 0.472 & & & & \\
\hline \multicolumn{8}{|c|}{ Multivariate logistic regression analysis } \\
\hline DM & 4.884 & $1.218 / 19.583$ & $0.025^{*}$ & J-CTO score & 15.882 & $1.959 / 134.631$ & $0.011^{*}$ \\
\hline
\end{tabular}

Data are expressed as mean \pm SD or number (\%)

BMI: body mass index, CKD: chronic kidney disease, DM: diabetes mellitus, $G(N)$ : normal LVEF group, $G(M)$ : mid-range LVEF group, $G(L)$ : low LVEF group, HTN: hypertension, ${ }^{*}$ :significant

group (N), MACCE occurred in 3 (12\%) of 25 patients ( 2 cases of NSTE-ACS with non-TVR and 1 case of stroke). In group (M), 4 (16\%) of 25 patients had MACCE (2 cases of decompensated heart failure needing hospitalization, 1 case of non-fatal MI with non-TVR, and 1 case of TVR). In group (L), MACCE were recorded in 7 (28\%) of 25 patients (4 cases of decompensated heart failure needing hospitalization, 1 case of non-fatal MI with non-TVR, 1 case of stroke and 1 case of malignant arrhythmia). There was no significant difference between the three groups regarding the reported MACCEs $\left(\chi^{2}=2.283\right.$ and $p>0.05$ ) (Fig. 3).

\section{Discussion}

The benefit and safety of CTO PCI in patients with low and mid-range LVEF continue to be of limited evidence. Successful CTO PCI was claimed to be associated with the improvement of cardiovascular outcome in comparison with unsuccessful procedures [18, 19]. However, myocardial revascularization guidelines still did not

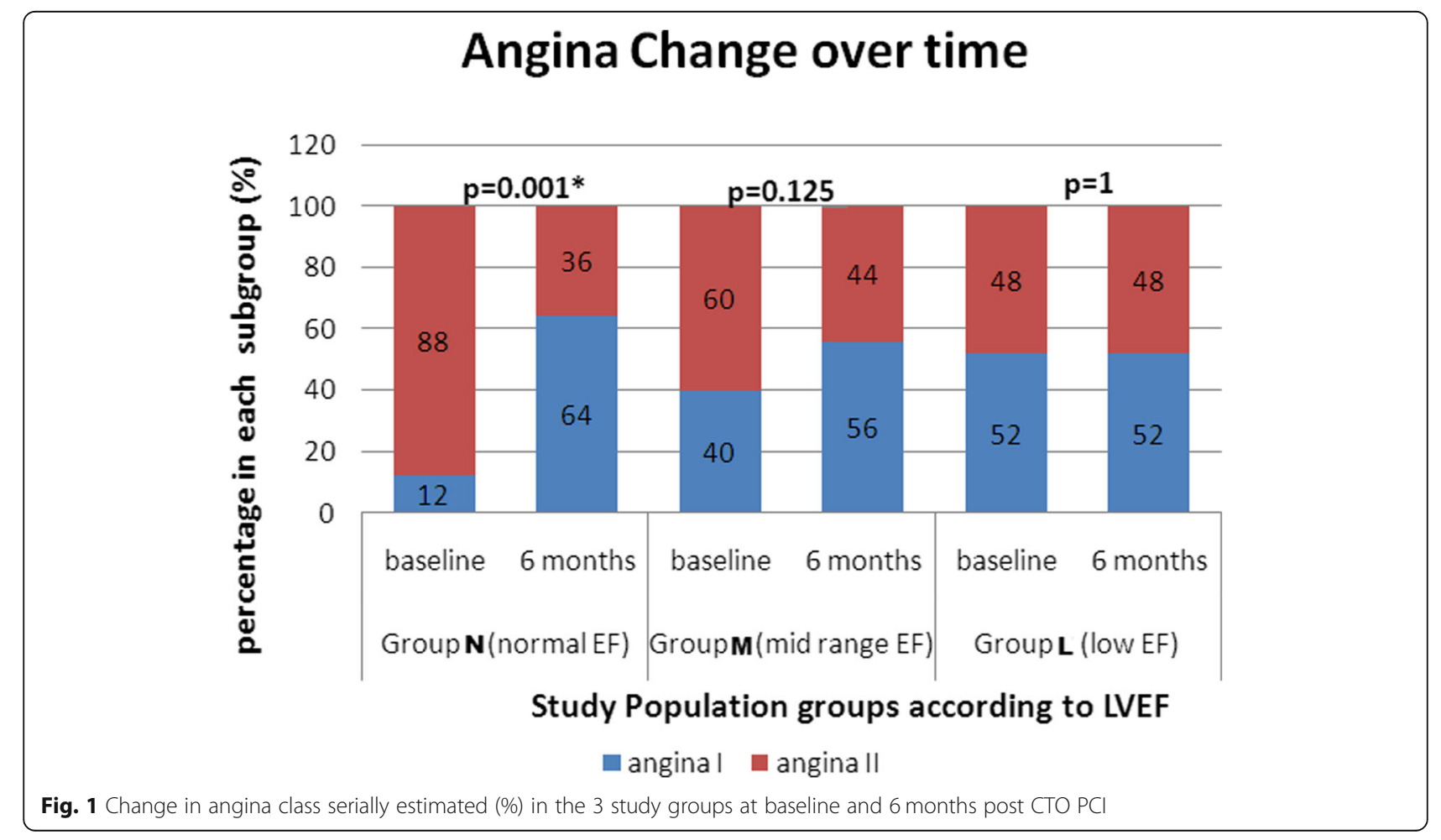




\section{Dyspnea Change over time}

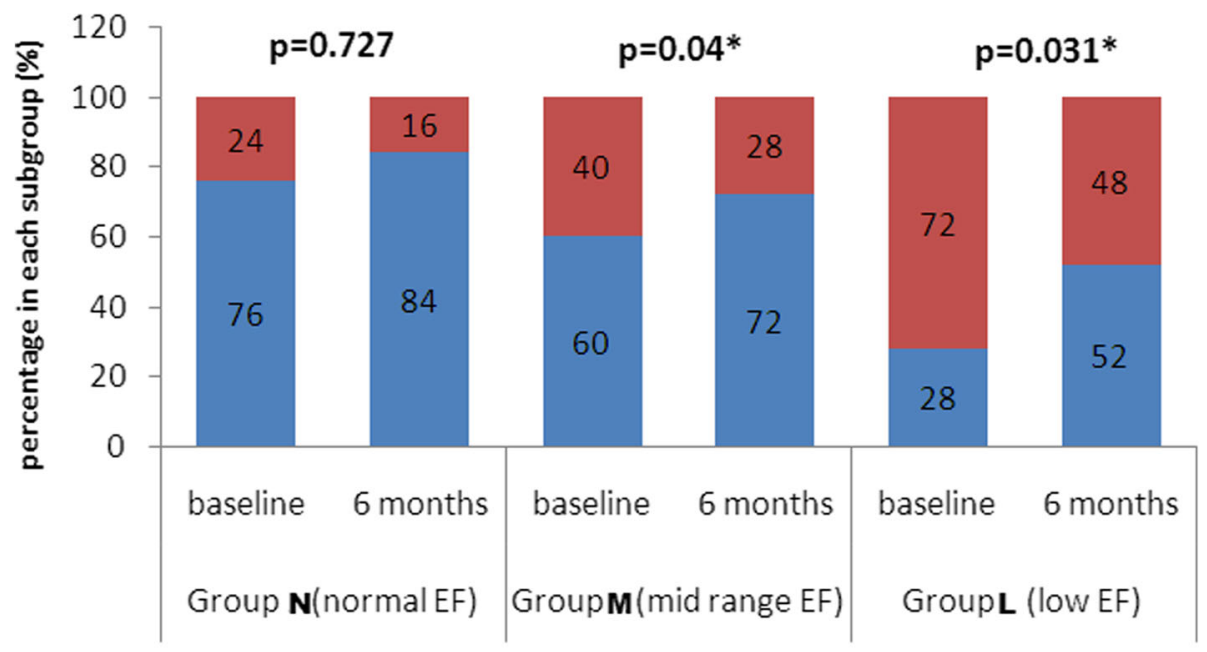

Study Population groups according to LVEF

\section{घyspneal a dyspneall}

Fig. 2 Change in dyspnea class serially estimated (\%) in the 3 study groups at baseline and 6 months post CTO PCI

address any solid indication for the most convenient management in patients with CTOs and impaired LVEF $[7,8]$.

Regarding baseline clinical characteristics, our data confirmed that the low LVEF group had more diabetic and CKD patients, compared with preserved and midrange LVEF groups. Moreover, patients having low
LVEF were more complaining of accelerated exertional dyspnea (NYHA $\geq 3$ ) compared to the other 2 groups. This finding is in concordance with Galassi et al. [20] and Tajstra et al. [6], who exhibited a higher prevalence of DM, CKD and peripheral vascular disease (PVD) in patients with CTO and low LVEF. Moreover, Tajstra et al. suggested that the presence of CTO in patients

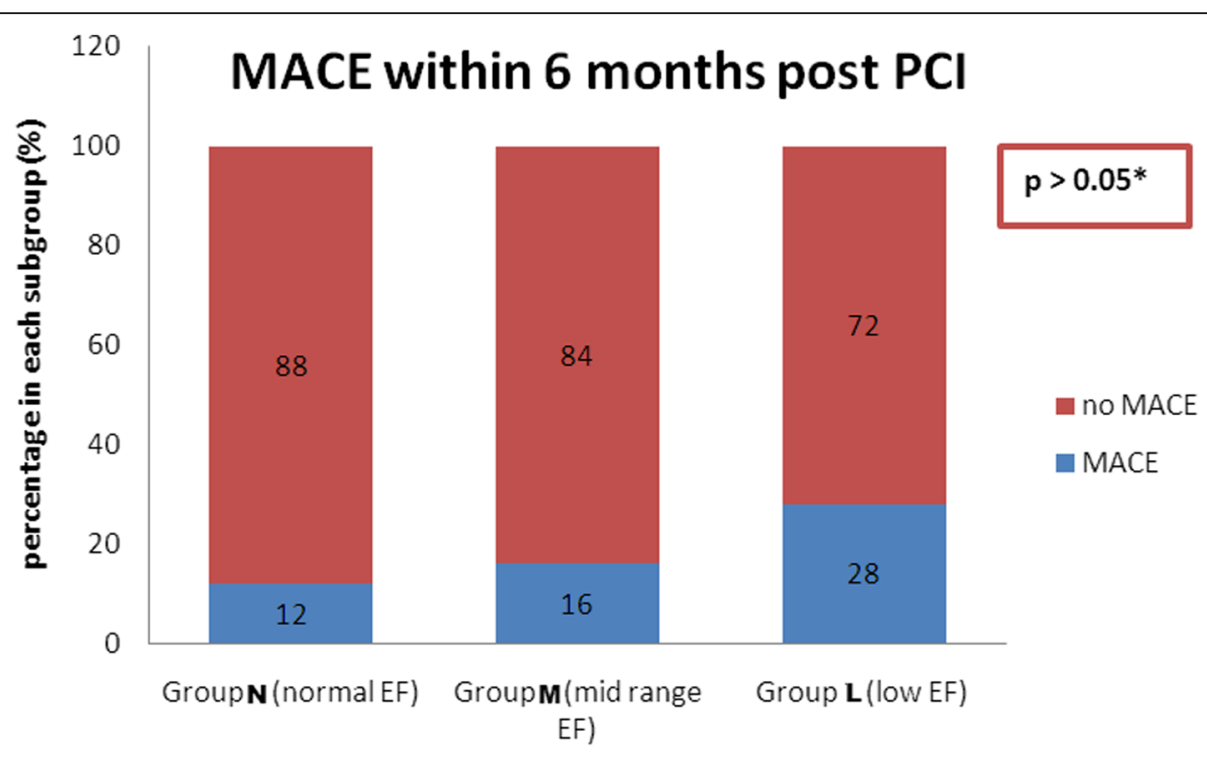

Study Population groups according to LVEF

Fig. 3 Major adverse cardiac events estimated (\%) in the 3 study groups within 6 months post CTO PCl 
with systolic heart failure was associated with significantly higher 12-month all-cause and cardiovascular deaths compared with patients without CTO. Actually, only $3.5 \%$ of patients of this study were affected by CTO PCI [6].

In our study, CTO PCI procedural success rate was $82.6 \%$ in all population, $76 \%$ in low LVEF group and $84 \%$ in mid-range LVEF group, with no significant increase of periprocedural complications when compared with normal LVEF group. Except for one case of coronary perforation with pericardial tamponade mandating pericardiocentesis, no patients with low or mid-range LVEFs suffered from severe periprocedural complications after CTO PCI. Moreover, impaired LVEF was not a predictor of CTO PCI periprocedural complications.

These results came hand in hand with Galassi et al. who revealed no significant correlation between impaired LVEF and periprocedural complications [20]. In the same context, Tajti et al. showed that despite CTO PCI in patients with < 35\% LVEF had lower technical success when compared with other two groups with 35$49 \%$ LVEF and > 50\% LVEF ( $83 \%$ vs. $88 \%$ and $89 \%, p=$ $0.03)$, yet with similar procedural success ( $82 \%$ vs. $85 \%$ and $86 \%, p=0.17)$ and major in-hospital complication rates $(3.7 \%$ vs. $2.9 \%$ and $2.4 \%, p=0.41)$ [21]. Similar results were recently reported by Danek et al. [22] with a low rate of periprocedural complications.

Although the CTO PCI techniques used in patients with low LVEF were similar to those performed in those with preserved and mid-range LVEF, the lowest amount of contrast should be considered, as 50\% of periprocedural complications recorded in this highrisk group were related to CIN. This could explain why contrast load was significantly lower in the low LVEF population of our study. Accordingly, our data propose that CTO PCI is a safe strategy when performed by experienced operators even in high-risk patients.

Successful CTO PCI was reported to confer benefit to patients over failed PCI [23]. Comparison of successful and failed CTO PCI done in a large meta-analysis showed a $44 \%$ reduction in mortality, a $55 \%$ reduction in residual angina and a $78 \%$ reduction in subsequent CABG in successful CTO PCI [24]. This mortality benefit can be attributed to many factors. Ischemia from the CTO territory may cause arrhythmic death. The presence of a CTO has been shown to be associated with higher rates of ventricular arrhythmia and all-cause death [25]. Furthermore, patients with CTOs cannot withstand an acute MI as multiple territories are simultaneously affected, what so-called double jeopardy [26].

It is generally accepted by consensus and guidelines that CTO PCI is indicated for symptomatic patients
[27]. Improvement in angina status after CTO-PCI has also been shown in the previous studies in patients with preserved LVEF [28, 29]. In the current study, a good clinical outcome was achieved in the 3 groups and impaired LVEF was not associated with increased incidence of MACCE at 6 months follow up. Moreover, there was a significant improvement in NYHA functional class in patients with low and mid-range LVEF, with significant improvement of CCS class in patients with preserved LVEF.

These results are concordant with Galassi and his colleagues, as low LVEF did not independently predict MACCE at mid-term follow-up. There was also a significantly less dyspnea in patients with low LVEF and less angina in those with preserved LVEF 6 months post CTO PCI, with mid-range LVEF population being still in the gray zone [20]

Another finding, from a clinical point of view; showed an improvement in angina and NYHA functional class, after CTO recanalization in patients with HFrEF, which could lead to improved prognosis in this population and provide a rationale for attempting CTO recanalization after viability and/ or ischemia confirmation in the CTO territory [30].

Moreover, in a study by Borgia et al, the Seattle Angina Questionnaire-UK version (SAQ-UK) was used to assess patients' angina quality of life after CTO PCI attempt. Compared to the unsuccessful group, the successful CTO PCI group reported fewer angina frequency and less physical limitation, and most were either asymptomatic or only CCS I [31]. Furthermore, Erdogan et al. showed that significant improvement of angina was achieved by successful CTO revascularization, where angina of CCS 2 or greater was improved in all patients who suffered from those degrees of angina prior to revascularization [32].

Needless to say that demonstration of myocardial viability is essential for improvement of survival and symptoms in patients with left ventricular dysfunction undergoing PCI regardless of the type of lesion [33].

Finally, what is really interesting in our study is being-up to our knowledge - the first study to evaluate the procedural consequences and clinical outcome post CTO PCI in such a peculiar category of mid-range LVEF (40-49\%). Our study showed significant improvement in symptoms 6 months post PCI with no significant increase in periprocedural or mid-term adverse events, compared to patients with low or preserved LVEFs. In fact, further studies are necessary to address clinical characteristics, procedural details and more long term outcomes in such specific category as well defined by current guidelines.

\section{Conclusion}

According to our study, CTO PCI represents an efficient and safe strategy in patients with mid-range LVEF (40- 
49\%) and low LVEF (< 40\%) affected by CTOs. In a 6 months follow-up period, significant improvement of mid-term clinical outcome, particularly dyspnea, was related to successful CTO PCI. Also, impaired LVEF categories were not associated with a higher risk of MACCE at follow-up.

\section{Limitations}

The first limitation of our study is being non-randomized study; therefore, changes in clinical parameters could not be specifically confirmed if being due to successful CTO PCI alone or related to optimal medical therapy. Second drawback was the relatively small sample size. Third, the short term follow-up period may alter the proper assessment of clinical outcomes, especially in those with impaired LVEF. Fourth, we did not perform a second angiography at 6 months after PCI in any of the patients. Finally, our study did not include CABG in patients with low and mid-range LVEFs for comparison versus PCI.

\section{Abbreviations}

2-D: Two dimensional; ACS: Acute coronary syndrome; AF: Atrial fibrillation; BMI: Body mass index; CABG: Coronary artery bypass grafting; CAD: Coronary artery disease; CCS: Canadian Cardiovascular Society; CIN: Contrast-induced nephropathy; CKD: Chronic kidney disease; CTO: Chronic total occlusion; DM: Diabetes mellitus; DSE: Dobutamine stress echocardiography; HFrEF: Heart failure with reduced ejection fraction; IHD: Ischemic heart disease; LVEF: Left ventricular ejection fraction; MACCE: Major adverse cardiac and cerebrovascular events; Nom.: Number; MI: Myocardial infarction; NYHA: New York Heart Association; PCl: Percutanous coronary intervention; PVD: Peripheral vascular disease; SAQ-UK: Seattle Angina Questionnaire-UK version; SD: Standard deviation; SPSS: Statistical Package for the Social Sciences; TIMI: Thrombolysis in myocardial infarction; TVR: Target vessel revascularization

\section{Acknowledgements}

This work was supported by the Cardiology Department in Zagazig University Hospitals, Cardiology Department in Kobry Al Kobbah Military Hospital and by my beloved family.

\section{Authors' contributions}

WSEA and MS have contributed to format the design of the study and revised the collected data, MMA has done the main work of the statistical data analysis, KAS has shared the operational work of the study and writing works, MaMA has recruited the participants, operational works, interpretation and analysis of the results. Finally, all authors have read, revised, and approved the manuscript.

\section{Funding}

No funding resources.

\section{Availability of data and materials}

Available by the corresponding author upon request

\section{Ethics approval and consent to participate}

This study was approved by the ethics committee of the Institutional Review Board (IRB) for medical research ethics, Faculty of Medicine, Zagazig University. A detailed informed verbal consent has been taken from all participants as it was just observational study with no intervention and they have to undergo CTO PCI anyway with our follow up that was of a great value for them and this consent was approved by the ethics committee. Ethics committee's reference number: ZU-IRB\#3943.

Consent for publication

Available in the same consent.

\section{Competing interests}

None of the authors have any relationships with companies or other financial conflicts of interest associated with the work in the manuscript.

\section{Author details}

${ }^{1}$ Cardiology Department, Faculty of Medicine, Zagazig University, Zagazig, Ash Sharkia, Egypt. ${ }^{2}$ Cardiology Department, Military Medical Academy, Cairo, Egypt.

Received: 1 November 2019 Accepted: 7 May 2020

Published online: 24 May 2020

\section{References}

1. Sianos G, Werner GS, Galassi AR, Papafaklis MI, Escaned J, Hildick-Smith D et al (2012) Recanalisation of chronic total coronary occlusions: 2012 consensus document from the EuroCTO club. Eurolntervention 8(1):139-145

2. Farooq V, Serruys PW, Garcia-Garcia HM, Zhang Y, Bourantas CV, Holmes DR et al (2013) The negative impact of incomplete angiographic revascularization on clinical outcomes and its association with total occlusions: the SYNTAX (Synergy Between Percutaneous Coronary Intervention with Taxus and Cardiac Surgery) Trial. J Am Coll Cardiol 61(3): 282-294

3. Tsai TT, Stanislawski MA, Shunk KA, Armstrong EJ, Grunwald GK, Schob AH et al (2017) Contemporary incidence, management, and long-term outcomes of percutaneous coronary interventions for chronic coronary artery total occlusions: insights from the VA CART program. JACC Cardiovasc Interv 10(9):866-875

4. Safley DM, Koshy S, Grantham JA, Bybee KA, House JA, Kennedy KF et al (2011) Changes in myocardial ischemic burden following percutaneous coronary intervention of chronic total occlusions. Catheter Cardiovasc Interv 78(3):337-343

5. Tio RA, Dabeshlim A, Siebelink HM, de Sutter J, Hillege HL, Zeebregts CJ et al (2009) Comparison between the prognostic value of left ventricular function and myocardial perfusion reserve in patients with ischemic heart disease. J Nucl Med 50(2):214-219

6. Tajstra M, Pyka Ł, Gorol J, Pres D, Gierlotka M, Gadula-Gacek E et al (2006). Impact of chronic total occlusion of the coronary artery on long-term prognosis in patients with ischemic systolic heart failure: insights from the COMMITHF registry. J Am Coll Cardiol Intv 9(17):1790-1797.

7. Windecker S, Kolh P, Alfonso F, Collet JP, Cremer J, Falk V et al (2014) ESC/ EACTS guidelines on myocardial revascularization: the task force on myocardial revascularization of the European Society of Cardiology (ESC) and the European Association for Cardio-Thoracic Surgery (EACTS). Developed with the special contribution of the European Association of Percutaneous Cardiovascular Interventions (EAPCI). Eur Heart J 35(37):2541-2619

8. Patel MR, Calhoon JH, Dehmer GJ, Smith PK, Spertus JA, Masoudi FA et al (2017) ACCF/SCAI/STS/AATS/AHA/ASNC/HFSA/SCCT 2017 appropriate use criteria for coronary revascularization in patients with stable ischemic heart disease: a report of the American College of Cardiology Appropriate Use Criteria Task Force, American Association for Thoracic Surgery, American Heart Association, American Society of Echocardiography, American Society of Nuclear Cardiology, Society for Cardiovascular Angiography and Interventions, Society of Cardiovascular Computed Tomography, and Society of Thoracic Surgeons. J Am Coll Cardiol 69(17):2212-2241

9. Campeau L (1976) Grading of angina pectoris. Circulation 54(3):522-523

10. Dolgin M, Association NYH, Fox AC, Gorlin R, Levin Rl, New York Heart Association. Criteria Committee. Nomenclature and criteria for diagnosis of diseases of the heart and great vessels. 9th ed. Boston, MA: Lippincott Williams and Wilkins; March 1, 1994

11. Schiller NB, Shah PB, Crawford M (1989) Recommendation for quantitation of the left ventricle by two-dimensional echocardiography. American Society of Echocardiography Committee on Standards, Subcommittee on Quantitation of two- Dimensional Echocardiogram. J Am Soc Echocardiogr 2(5):358-367

12. Lang RM, Badano LP, Mor-Avi V, Afilalo J, Armstrong A, Ernadel L et al (2015) Recommendations for cardiac chamber quantification by echocardiography in adults: An update from the American Society of Echocardiography and the European Association of Cardiovascular Imaging. J Am Soc Echocardiogr 28(1):1-31

13. Morino $Y$, Abe M, Morimoto $T$, Kimura T, Hayashi $Y$, Muramatsu T et al (2011) J-CTO registry investigators. predicting successful guidewire crossing 
through chronic total occlusion of native coronary lesions within 30 minutes: the J-CTO (Multicenter CTO Registry in Japan) score as a difficulty grading and time assessment tool. JACC Cardiovasc Interv 4(2):213-221

14. Sianos G, Morel MA, Kappetein AP, Morice MC, Colombo A, Dawkins K et a (2005) The SYNTAX Score: an angiographic tool grading the complexity of coronary artery disease. Eurolntervention 1(2):219-227

15. TIMI Study Group (1985) The thrombolysis in myocardial infarction (TIMI) trial. Phase I findings. N Engl J Med 312(14):932-936

16. Kern MJ, Moore JA, Aguirre FV, Bach RG, Caracciolo EA, Wolford T et al (1996) Determination of angiographic (TIMI Grade) blood flow by intracoronary doppler flow velocity during acute myocardial infarction. Circulation 94(7):1545-1552

17. Chesebro JH, Knatterud G, Roberts R, Borer J, Cohen LS, Dalen J et al (1987) Thrombolysis in myocardial infarction (TIMI) trial, phase l: a comparison between intravenous tissue plasminogen activator and intravenous streptokinase. Clinical findings through hospital discharge. Circulation 76(1): 142-154

18. Hoebers LP, Claessen BE, Elias J, Dangas GD, Mehran R, Henriques JP (2015) Meta-analysis on the impact of percutaneous coronary intervention of chronic total occlusions on left ventricular function and clinical outcome. Int J Cardiol 187:90-96

19. George S, Cockburn J, Clayton TC, Ludman P, Cotton J, Spratt J et al (2014) Longterm follow-up of elective chronic total coronary occlusion angioplasty: analysis from the U.K. Central Cardiac Audit Database. J Am Coll Cardiol 64(3):235-243

20. Galassi AR, Boukhris M, Toma A, Elhadj Z, Laroussi L, Gaemperli O et al (2017) Percutaneous coronary intervention of chronic total occlusions in patients with low left ventricular ejection fraction.JACC. Cardiovasc Interv Ther 10(21):2158-2170

21. Tajti P, Karmpaliotis D, Alaswad K, Jaffer F, Yeh RW, Patel M et al (2019) Procedural outcomes of percutaneous coronary interventions for chronic total occlusions in patients with low left ventricular ejection fraction: insights from the progress CTO registry. J Am Coll Cardiol Interv 73(9):1279

22. Danek BA, Karatasakis A, Karmpaliotis D, Alaswad K, Yeh RW, Jaffer F et a (2016) Development and validation of a scoring system for predicting periprocedural complications during percutaneous coronary interventions of chronic total occlusions: the prospective global registry for the study of chronic total occlusion intervention (PROGRESS CTO) complications score. J Am Heart Assoc 5(10):e004272

23. Galassi AR, Sianos G, Werner GS, Escaned J, Tomasello SD, Boukhris M et al (2015) Retrograde recanalization of chronic total occlusions in Europe. Procedural, in-hospital, and long-term outcomes from the multicenter ERCTO registry. J Am Coll Cardiol 65(22):2388-2400

24. Joyal D, Afilalo J, Rinfert S (2010) Effectiveness of recanalization of chronic total occlusions: a systematic review and metaanalysis. Am Heart J 160(1): 179-187

25. Nombela-Franco L, Mitroi CD, Fernandez-Lozano I, García-Touchard A, Toquero J, Castro-Urda V et al (2012) Ventricular arrhythmias among implantable cardioverter defibrillator recipients for primary prevention: impact of chronic total coronary occlusion (VACTO Primary Study). Circ Arrhythm Electrophysiol 5(1):147-154

26. Claessen BE, Dangas GD, Weisz G, Witzenbichler B, Guagliumi G, Möckel M et al (2012) Prognostic impact of a chronic total occlusion in a non-infarctrelated artery in patients with ST-segment elevation myocardial infarction: 3-year results from the HORIZONS-AMI trial. Eur Heart J 33(6):768-775

27. Brilakis ES, Banerjee S, Karmpaliotis D, Lombardi WL, Tsai TT, Shunk KA et al (2015) Procedural outcomes of chronic total occlusion percutaneous coronary intervention: a report from the NCDR (National Cardiovascular Data Registry). JACC Cardiovasc Interv 8(2):245-253

28. Bucciarelli-Ducci C, Auger D, Di Mario C, Locca D, Petryka J, O'Hanlon R et al (2016) CMR guidance for recanalization of coronary chronic total occlusion. J Am Coll Cardiol Intv 9(5):547-556

29. Christakopoulos GE, Christopoulos G, Carlino M, Jeroudi OM, Roesle M, Rangan BV et al (2015) Meta-analysis of clinical outcomes of patients who underwent percutaneous coronary interventions for chronic total occlusions. Am J Cardiol 115(10):1367-1375

30. Cardona M, Martín V, Prat-Gonzalez S, Ortiz JT, Perea RJ, de Caralt TM et al (2015) Benefits of chronic total coronary occlusion percutaneous intervention in patients with heart failure and reduced ejection fraction: insights from a cardiovascular magnetic resonance study. J Cardiovasc Magn Reson 18:78
31. Borgia F, Viceconte N, Ali O, Stuart-Buttle C, Saraswathyamma A, Parisi R et al (2012) Improved cardiac survival, freedom from MACE and anginarelated quality of life after successful percutaneous recanalization of coronary artery chronic total occlusions. Int J Cardiol 161(1):31-38

32. Erdogan E, Akkaya M, Bacaksiz A, Tasal A, Sönmez O, Elbey MA et al (2013) Early assessment of percutaneous coronary interventions for chronic total occlusions analyzed by novel echocardiographic techniques. Clinics 68(10): 1333-1337

33. Gerber BL, Rousseau MF, Ahn SA, le Polain de Waroux JB, Pouleur AC, Phlips T et al (2012) Prognostic value of myocardial viability by delayed-enhanced magnetic resonance in patients with coronary artery disease and low ejection fraction: impact of revascularization therapy. J Am Coll Cardiol 59(9):825-835

\section{Publisher's Note}

Springer Nature remains neutral with regard to jurisdictional claims in published maps and institutional affiliations.

\section{Submit your manuscript to a SpringerOpen ${ }^{\circ}$ journal and benefit from:}

- Convenient online submission

- Rigorous peer review

- Open access: articles freely available online

- High visibility within the field

- Retaining the copyright to your article

Submit your next manuscript at $\boldsymbol{\nabla}$ springeropen.com 